\title{
Biocompatibility assessment of biomaterials used in orthopedic devices: An overview (Review)
}

\author{
BOGDAN HUZUM ${ }^{1,2}$, BOGDAN PUHA ${ }^{2,3^{*}}$, RIANA MARIA NECOARA ${ }^{* *}$, STEFAN GHEORGHEVICI $^{2}$, \\ GABRIELA PUHA ${ }^{2,5}$, ALEXANDRU FILIP ${ }^{3}$, PAUL DAN SIRBU $^{3}$ and OVIDIU ALEXA ${ }^{2,3}$ \\ ${ }^{1}$ Department of Physiology, Faculty of Medicine, 'Grigore T. Popa' University of Medicine and Pharmacy of Iasi, \\ 700115 Iasi; ${ }^{2}$ Orthopaedic and Traumatology Clinic, ‘Sf. Spiridon’ Clinical Emergency Hospital, 700111 Iasi; \\ ${ }^{3}$ Department of Orthopaedic and Traumatology, Faculty of Medicine, 'Grigore T. Popa' University of \\ Medicine and Pharmacy of Iasi, 700115 Iasi; ${ }^{4}$ Radiology-Imaging Clinic, 'Sf. Spiridon' Clinical Emergency Hospital, \\ 700111 Iasi; ${ }^{5}$ Department of Internal Medicine and Toxicology, Faculty of Medicine, \\ 'Grigore T. Popa' University of Medicine and Pharmacy of Iasi, 700115 Iasi, Romania
}

Received April 29, 2021; Accepted May 31, 2021

DOI: $10.3892 /$ etm.2021.10750

\begin{abstract}
Biocompatibility is one of the mandatory requirements for the clinical use of biomaterials in orthopedics. It refers to the ability of a biomaterial to perform its function without eliciting toxic or injurious effects on biological systems but producing an appropriate host response in a specific case. Today, the biocompatibility concept includes not only bio-inertia, but also biofunctionality and biostability. High biocompatibility and functional properties are highly desirable for new biomaterials. The chemical, mechanical, structural properties of biomaterials, their interaction with biological environment or even the methodology of assessment can influence the biocompatibility. The biological evaluation of biomaterials includes a broad spectrum of in vitro and in vivo tests related to the cytocompatibility, genotoxicity, sensitization, irritation, acute and chronic toxicity, hemocompatibility, reproductive and developmental toxicitity, carcinogenicity, implantation and degradation as specified in different international standards. A brief review of the main assays used in the biocompatibility testing of orthopedic biomaterials is presented. In addition, their main biocompatibility issues are overviewed.
\end{abstract}

Correspondence to: Dr Bogdan Puha, Department of Orthopaedic and Traumatology, Faculty of Medicine, 'Grigore T. Popa' University of Medicine and Pharmacy of Iasi, 16 Universitatii Street, 700115 Iasi, Romania

E-mail:puhab@yahoo.com

${ }^{*}$ Contributed equally

Key words: biocompatibility, evaluation, biomaterials, orthopedics, biofunctionality

\section{Contents}

1. Introduction

2. Biomaterials in orthopedics

3. Biocompatibility testing

4. Conclusions

\section{Introduction}

Orthopedic injuries and associated pathologies are an important public health issue worldwide as well as a major global burden of disability and suffering. It is estimated that the musculoskeletal conditions affect approximately 1.71 billion individuals globally (1). The Global Burden of Disease, Injuries, and Risk Factors Study (GBD) 2019 reported for the 1990-2019 time period musculoskeletal disorders as one of the 10 significant causes of enhancing burden from teenage years to older age with a $30.7 \%$ increase in DALY rates (Disability-Adjusted Life-Years) (2). These conditions or orthopedic trauma often require surgery and the use of permanent, temporary or biodegradable medical devices that include various natural or synthetic biomaterials able to substitute or repair different tissues (ligaments, tendons, cartilage, bone). The design of the medical device, functional properties of biomaterials and the bioresponse are some of the key players that determine the clinical success of the orthopedic intervention. Apart from their mechanical, microstructural and chemical characteristics, biomaterials are screened for their innocuity, biocompatibility, safety and efficacy for the clinical efficacy (3).

Biocompatibility, one of the most important features of biomaterials, is defined as 'the ability of a biomaterial to perform its desired function with respect to a medical therapy, without eliciting any undesirable local or systemic effects on the recipient or beneficiary of that therapy, but meanwhile generating the most optimized clinically relevant performance of that therapy' or 'the ability of a material to perform with an 
appropriate host response in a specific situation' $(3,4)$. Thus, biocompatibility testing is a primary requirement in the development and the approval of orthopedic materials for clinical use by regulatory agencies. Biomaterials need to meet basic biocompatibility criteria as set by the International Standards Organization (ISO 10993). They must be nontoxic, nonthrombogenic, noncarcinogenic, nonantigenic and nonmutagenic in order to exhibit an appropriate biological response (5).

In this regard, biocompatibility testing is a complex process that include in vitro and in vivo specific tests depending on the end-use application of the biomaterials. The goal of this review is to provide a roadmap for the practical approach to the biocompatibility testing for orthopedic materials. The most important assays in this area are discussed based on the current findings. In addition, the review summarizes the main categories of biomaterials in orthopedics and their biocompatibility issues.

\section{Biomaterials in orthopedics}

According to the American National Institute of Health, a biomaterial is 'any substance or combination of substances, other than drugs, synthetic or natural in origin, which can be used for any period of time, which augments or replaces partially or totally any tissue, organ or function of body in order to maintain or improve the quality of life of the individual' (6). The main required properties of orthopedic biomaterials are good mechanical abilities including high resistance to corrosion and wear, biocompatibility, chemical stability and appropriate microstructural characteristics (Fig. 1) (7,8). A high longevity of materials expressed by unaltered properties after a long time of contact with the biological surroundings are tremendously essential.

Depending on their nature, orthopedic materials can be classified as metals, ceramics, polymers and composites.

Metallic materials. Due to their superior mechanical properties, metals have been mostly used in prostheses stems, fracture plates, or as load-bearing components in total joint replacement. The most commonly used metals include stainless steels (316L), titanium and titanium-base alloys (Ti-6Al-4V, TiAl4VELI, Ti6Al17Nb) and cobalt alloys (Co-Cr-Mo) (Table I) (7-9). Among these, titanium and its alloys show an excellent corrosion resistance and a high biocompatibility as well as good long-term behavior (10). In addition, Co-Cr based alloys exhibit good mechanical properties including excellent wear resistance, but they have a low biocompatibility (11). The main biocompatibility issues related to metallic materials and their alloys are bioactivity, corrosion byproducts, hypersensitivity reactions (mostly in the case of nickel alloys), and lipid uptake (5).

The coating of metals with bioactive ceramics and the chemical modification of metal surface by binding of polymers and biomolecules allow the biofunctionalization of metallic materials and the control of the biodegradability rate and biocompatibility $(8,12)$. In this regard, magnesium alloys (Mg-Ca, Mg-Zn) and magnesium matrix composites (Mg-calcium phosphate particle, $\mathrm{Mg}$-hydroxyapatite, Mg-tricalcium phosphate) are one of the metallic materials which have recently attracted growing interest due to their improved mechanical and biological properties $(9,13)$. Magnesium is ideal for biodegradable orthopedic implants owing to its high biocompatibility, osteogenesis ability and biodegradable behavior (14).

Ceramics. Ceramics represent a family of inorganic/non-metallic products with a broad range of composition that can be dense and resorbable like tricalcium phosphate or dense, non-porous and chemically binding to the bone such as hydroxyapatite (HA) $(15,16)$. They are widely used in orthopedic applications as bone replacement in hip and knee reconstruction. The main advantages of these materials are high corrosion resistance, hardness, wear resistance, low friction, significant biocompatibility and osseointegration with the host tissue. On the basis of tissue-material interface reaction, bioceramics are generally classified into three categories: i) bioactive (hydroxyapatite, bioactive glasses); ii) bioresorbable (calcium phosphate) and iii) bioinert (alumina, zirconia) (Table I) $(8,9,17-20)$. It is of interest to modulate the chemistry of ceramics that they become osteoinductive but also to enhance the bone regeneration rate. The biocompatibility issues that could be considered in the ceramics category are related to ADME profile (adsorption, deposition, excretion, and metabolism), bioactivity, lipid uptake and thromboresistance (5).

Polymers. Some of the most known polymers of first generation are acrylic resins, polyethylene (PE) and ultrahigh molecular weight PE (UHMWPE) and polymethacrylate (PMMA) (Table I) $(11,12,19,21-23)$. They are characterized by structural stability, low cost but a relative biocompatibility. Their main use refers to joint replacement, anchorage of prostheses and hip arthroplasties. The second generation of polymers are biodegradable and resorbable materials that are of interest in orthopedic practice for the purpose of bone substitution, and repair of bone fractures, cartilage, or membranes. Some examples of biodegradable polymers include polyglicolide (PGA), polylactide (PLA), polydioxanone (PDS), poly(e-caprolactone) (PCL) or chitosan $(5,11)$. The biocompatibility of polymeric materials is influenced by various factors as structural properties (chemical structure, molecular weight, functional groups, hydrophobicity), surface morphology, wetting abilities, or electrical charge. Thus, the increase in molecular weight of polyethylene glycol is associated with a decrease in protein adsorption (9). The biocompatibility issues associated with these biomaterials differ slightly between the two polymer types; thus, in the case of synthetic polymers, these issues refer to the calcification, extractables, hypersensitivity reactions, lipid uptake, protein adsorption and sterilization residuals. For the biodegradable polymers, the biodegradation products, ADME, effect of infection (acid $\mathrm{pH}$ on biodegradation particulates), effect of hematoma (alkaline $\mathrm{pH}$ ) are the specific biocompatibility issues (5).

Composite biomaterials. Composites comprise at least two components, namely matrix material and a filler (reinforcement) and they are widely used in structural and automotive applications. The addition of the filler enhances structural properties, biocompatibility and bioactivity of the matrix. These biomaterials have been classified into metal matrix composites 


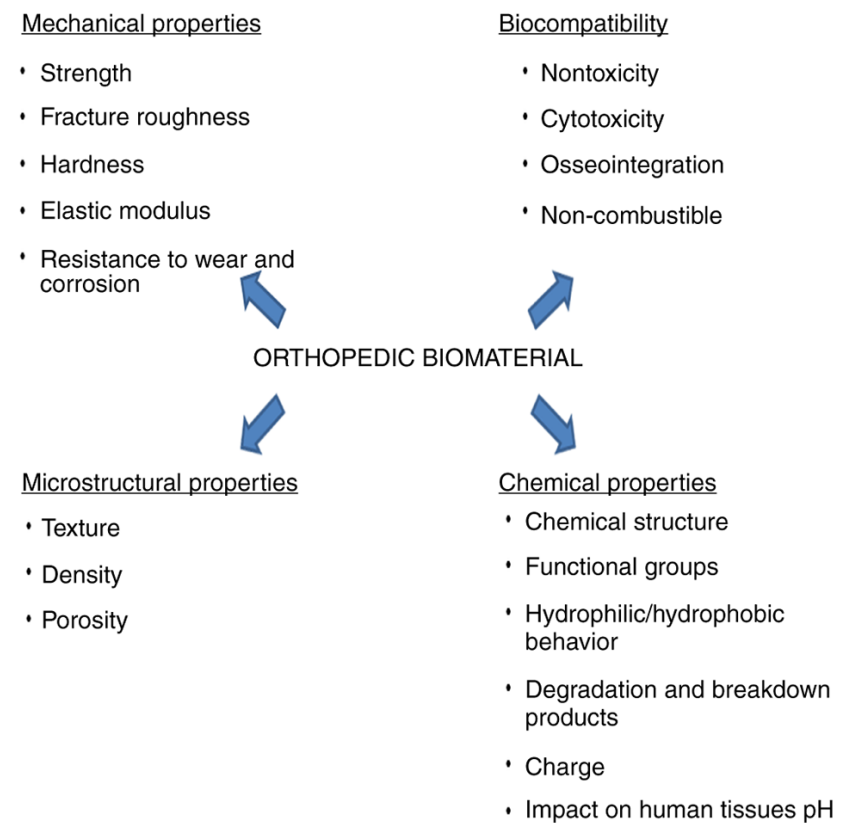

Figure 1. Essential characteristics of biomaterials in orthopedics $(7,8)$.

(Ti/HA, Mg/HA, Ti6Al4V/HA), ceramic matrix composites (HA/stainless steel) and polymer matrix composites (high density polyethylene/HA, carbon fiber/polyether ether ketone). Fiber-reinforced polymers and PMMA-composites are largely used in orthopedic devices $(7,9,16,17)$. The major biocompatibility issues described for composites include hypersensitivity, lipid uptake, matching tissue biomechanics, and surface exposure of compounded particles (5).

\section{Biocompatibility testing}

The designed materials for orthopedic uses should be capable to function in vivo without exhibiting any undesirable local or systemic effects as immune, allergic, inflammatory and carcinogenic responses (5). Biocompatibility includes not only bio-inertia, but also biofunctionality and biostability. It is a key concept that strongly depends on the material properties (texture, crystallinity, wettability, surface chemistry, breakdown products, charges, stiffness), interaction with the biological environment of targeted tissues (adsorption of proteins, inflammatory processes, contact with blood), period of the device application, and type of application (24-26).

Taking all this into account, the biological evaluation of biomaterials includes a broad spectrum of in vitro and in vivo tests related to the cytocompatibility, genotoxicity, sensitization, irritation, acute and chronic toxicity, hemocompatibility, reproductive and developmental toxicity, carcinogenicity, implantation and degradation as specified in different international standards (Table II) (3).

Before biocompatibility testing, the biomaterials from a final form of the medical device, are extracted using a semiphysiological medium (saline solution, cottonseed or sesame seed oil) or cell culture medium in small tubes. Then, they are usually incubated for $24-72 \mathrm{~h}$ at $37^{\circ} \mathrm{C}$. Furthermore, the resulting extract solutions are decanted into sterile glass tubes and used in the biological tests (3). The extraction procedure is very gentle. Only some compounds from the surface of materials can be extracted. Its main limitations include low extraction yield $(<10 \%)$, short-time contact between material and cells/tissue fluids compared to the in vivo conditions, and unsuitable extraction medium for organic hydrophobic compounds. The use of the extraction vehicle with low concentrations of dimethylsulphoxide $(<0.5 \%)$ allows solubilization of both hydrophilic and hydrophobic compounds (4).

Cytocompatibility tests. Cytocompatibility tests evaluate the biological reactivity of living cells to the biomaterial extract solutions including cell viability, growth, and metabolic activity (4). Toxic agents derived from biomaterials such as metal ions, reactive agents, residual monomers, may exert toxic effects on cell functions and viability. The cellular damage involves structural disintegration and alteration of cell morphology, reduction in cell adhesion and proliferation, decrease in metabolic activity and cell lysis (27).

Cytotoxicity elution test. Cytotoxicity elution test (MEM elution) is an in vitro qualitative assay. It involves the incubation of L-929 mouse fibroblast cells with an extract of the test material for $48 \mathrm{~h}$. After incubation, the cells are microscopically examined in terms of morphological changes (deformed and lysed cells). The cell responses to material are scored on a scale of 0 to 4 . The biomaterial is considered biocompatible if the cell response to the test material is not greater than grade 2 (mild reactivity) (3).

MTT assay. MTT assay is the most commonly used test to evaluate cell viability and proliferation. It relies on the enzymatic reduction of yellow 3-(4,5-dimethylthiazol-2-yl)-2,5-diphenyl-2H-tetrazolium bromide (MTT) to purple formazan in metabolically active cells. The color intensity of formed formazan is directly proportional to the number of viable cells. The measuring absorbance of formazan at $570 \mathrm{~nm}$ allows quantitative evaluation of living cells. The material is considered cytocompatible if the percentage of viable cells is equal to or higher than $70 \%(3,28)$. Although MTT is considered the 'gold standard' in cytotoxicity studies, the test has several limitations. Thus, the cell culture conditions may affect the metabolism and rate of MTT reduction, and the interaction between test material and MTT can modify the final results. The carbon nanotubes as well as calcium phosphate scaffolds are able to reduce MTT. In addition, carbon nanotubes can bind the formed formazan altering the test outcome $(4,28)$.

Agar overlay assay. Agar overlay assay is a qualitative test that evaluates cytotoxicity by indirect contact. Subconfluent cell cultures (mouse fibroblasts L929, NIH 3T3) are overlaid by an agar layer. The test material is placed on the agar layer and after 24-72 $\mathrm{h}$ exposure time, it is removed. Then, the cells under and around the test material are exposed to Neutral Red (3-amino-7-dimethylamino-2-methylphenazine hydrochloride) staining. The detachment, vacuolization and lysis of cells can be semi-quantitatively scored. Only the living cells accumulate the Neutral Red dye and they appear red colored to light-microscope analysis. The agar overlay assay is suitable for high density biomaterials. The limits of the test are related to the short time exposure to biomaterial (only acute cytotoxicity 


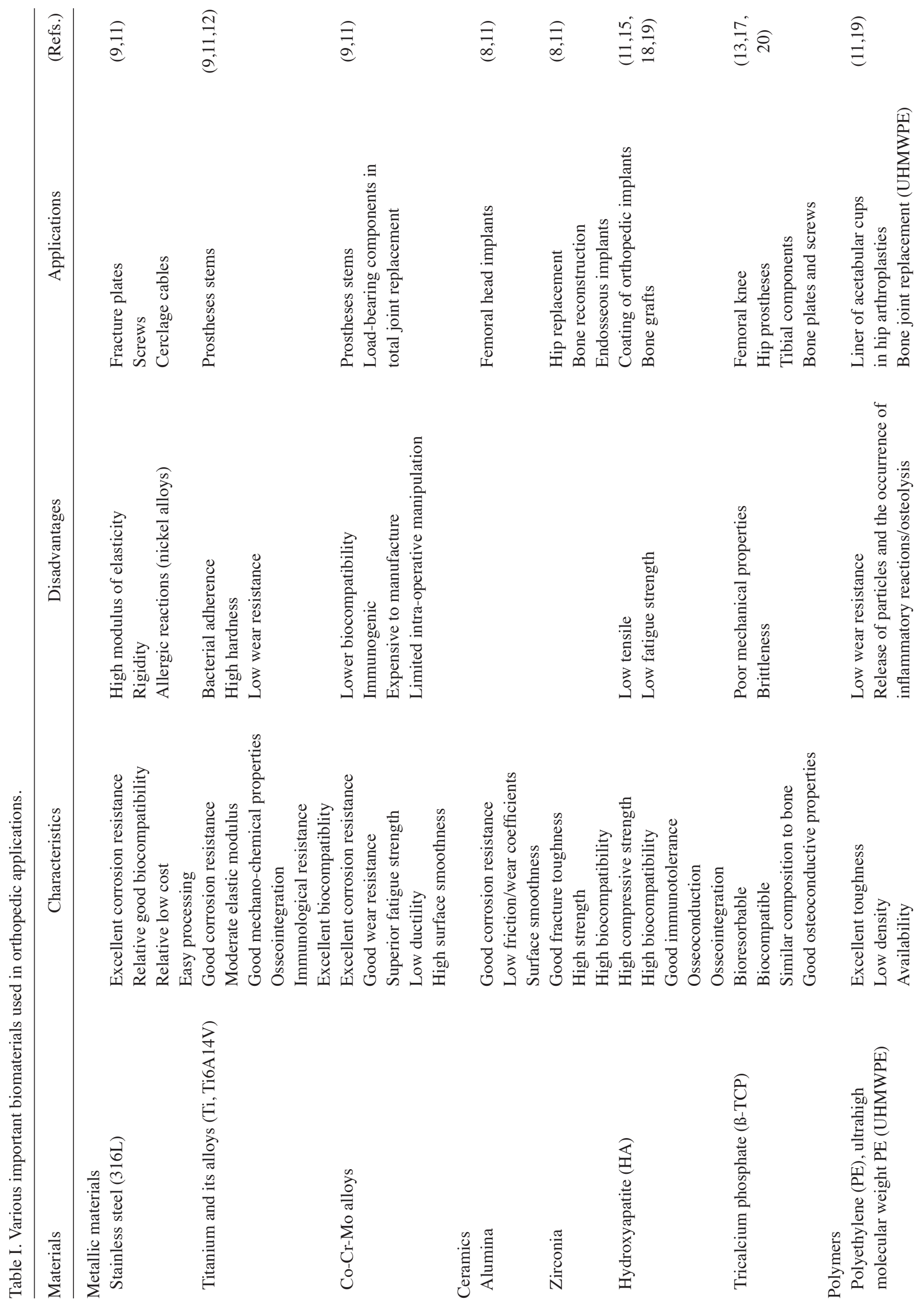


detection) and the binding or adsorption of Neutral Red on test or released compounds $(3,4,29)$.

Genotoxicity evaluation. Genotoxicity evaluation is an essential part of the safety assessment of biomaterials as damage of genetic material may result in the induction of carcinogenesis or may alter the reproductive function if germ cell DNA is impaired. The sensitive genotoxicity endpoints comprise DNA damage, gene mutations and chromosomal damage $(30,31)$. The genotoxic risk assessment of biomaterials is performed in mammalian and non-mammalian systems (3).

Mouse lymphoma assay. Mouse lymphoma assay (MLN) is an in vitro mammalian cell gene mutation test that is able to detect both gene mutations (point mutations) and clastogenic injuries (deletions, translocations, mitotic recombination/gene conversion, and aneuploidy). Basically, the test quantifies alterations of thymidine kinase $(T K)$ gene expression located on chromosome 11. The L5178YTK ${ }^{+/}-3.7 .2 \mathrm{C}$ mouse lymphoma cell line is used for the assay. The cells deficient in the $T K$ gene as a result of the mutation $\mathrm{TK}^{+/}$to $\mathrm{TK}^{-/}$induced by a genotoxicant are resistant to cytostatic activity of pyrimidine analogue triflurothymidine (TFT). In the presence of TFT in the medium, the mutant cells are able to survive and proliferate whereas the normal cells are not. Cells are incubated with test material for 3 to $4 \mathrm{~h}$ in the presence and absence on an metabolic activation system. In addition, the cells are exposed to test agent for $24 \mathrm{~h}$ without exogenous metabolic activation. A co-factor-supplemented post-mitochondrial fraction obtained from hepatic tissue of rodents is used for metabolic activation. After treatments, the cells are subcultured for two days to allow expression of the mutant phenotype. Then, the cells are seeded into 96-well plates with and without TFF to detect mutant cells and to evaluate cloning efficiency. Previously, the dose range of the test material is established depending on the cytotoxicity assessment. The occurrence of mutant colonies (small and large colonies) and the increase in mutant frequency (more than Global Evaluation Factor, 126x $10^{-6}$ ) is associated with the induction of chromosomal aberrations and the mutagenicity of the test material. The outcome of the test is significantly influenced by the cell line viability per se, solubilization of the test material, changes in the $\mathrm{pH}$, osmolality, and high levels of cytotoxicity $(3,32,33)$.

In vitro chromosomal aberration assay. In vitro chromosomal aberration assay evaluates structural aberrations (chromosome/chromatid breaks; chromosome/chromatid exchanges) but it also detects polyploidy and endoreduplication. The test is performed in primary human peripheral blood lymphocytes or in established cell lines such as Chinese hamster ovary (CHO) cells or Chinese hamster fibroblasts. In this method, the proliferating cells are exposed to the extract test dilutions with and without metabolic activation for 3 to $6 \mathrm{~h}$. The same metabolic activation system as in the MLA test is used. After exposure to the test material, the cell cultures are treated for $1-3 \mathrm{~h}$ with a metaphase-arresting compound (colcemid) at predetermined time intervals in relation with cell cycle length. The cells are harvested for the preparation of chromosomes by hypotonic treatment, fixation and staining with Giemsa. Chromosomal aberrations (number and types) in the mitotic cells are scored. 
Table II. International standards for biocompatibility testing of biomaterials $(4,5)$.

Standards ${ }^{\mathrm{a}}$

Biological response

ISO

ASTM

Cytotoxicity
Sensitization
Irritation
Acute systemic toxicity
Subacute toxicity
Genotoxicity
Immunoresponsiveness
Hemocompatibility
Chronic toxicity
Carcinogenicity
Degradation

Implantation
10993-5
10993-10
10993-10
10993-11
10993-11
10993-3
10993-20
10993-4
10993-11
10993-3
10993-6

10993-9; 10993-13

10993-14; 10993-15

F813-07; F895-84; F1027-06
F720-81; F2147-01; F2148-07
F719-81; F749-98
F750-87
-
E1262-88
F1906-98
F756-08
-
F1439-03
F1983-14

F1408-97; F763-04; F1904-98

F981-04; F1983-99

aSO, International Standards Organization; ASTM, American Society for Testing and Materials.

The percentage of cells with this chromosomal damage is evaluated for test material dilutions as compared to the value of the negative controls. Test conditions ( $\mathrm{pH}$ changes, osmolality) may influence the final results leading to a positive outcome. In addition, established cell lines should be characterized by stability in the modal chromosome number. The solvent used for the extraction of biomaterial should not interfere with the cell response $(3,34,35)$.

Reverse mutation assay. Reverse mutation assay (Ames test) is a nonmammalian test system that use mutagenicity in bacteria as an end point. The assay is performed with strains of Salmonella typhimurium and Escherichia coli that have point mutations in the genes of the histidine and tryptophan operon, respectively. The exposure of mutant bacterial cells to a mutagenic agent causes a reversal of initial mutation (back mutation) restoring the ability of the bacteria to grow on the media lacking in histidine. Bacterial cultures are exposed to the biomaterial in the presence and the absence of metabolic activation system, using either plate incorporation or preincubation before plating. After 2 to 3 days of incubation at $37^{\circ} \mathrm{C}$, the revertant colonies are counted compared to the control plates. An increased number of revertant colonies indicates the mutagenic potential of the tested material. The preincubation method is recommended for some materials such as divalent metals $(3,36,37)$.

Irritation (intracutaneous reactivity) testing. Irritation (intracutaneous reactivity) testing is an in vivo assay that evaluates the potential of biomaterials to cause irritation on the exposed area of the body. Saline and vegetable biomaterial extracts are administered by intracutaneous injection into multiple sites on the back of albino rabbits. The skin reactions (erythema, edema, scabbing, bleeding) are evaluated 24,48 and $72 \mathrm{~h}$ following injection using a standardized scoring scheme. The biomaterials meets the criteria of the test if the difference between its average irritation score and the value of control is 1 or $<1$. The use of rancid vegetable oil as a vehicle for biomaterials could determine an extreme reactivity hiding the true effect of the biomaterial $(3,38)$.

Skin sensitization assay. Skin sensitization assay evaluates the allergenic potential of biomaterials. Guinea pig maximization test is the most commonly used assay. It is based on the induction of an immune response of the skin and it comprises 3 phases. Stage I of the test (Induction) implies an intradermal injection of the biomaterial extract in guinea pigs on the test area. Then, a topical patch is applied after 7 days (Phase II, Induction II) and 14 days (Phase III, Challenge). Skin reactions (erythema, swelling) are evaluated at 24 and $48 \mathrm{~h}$ following patch removal using a scoring system. Sensitization potential is associated with a score value of 1 or greater in the test group $(3,39)$.

Acute systemic toxicity testing. Acute systemic toxicity testing estimates the hazard potential of a biomaterial following short-time exposure in animals. The extracts of the test material and negative controls are injected intravenously (saline extract) or intraperitoneally (cottonseed oil extract) into Swiss Albino mice. Then, the animals are observed 4, 24, 48, and $72 \mathrm{~h}$ after treatment. The body weight, survival, and animal behavior are recorded. The biomaterial meets the test requirements if its biological reaction is lower than the negative control (3).

Hemocompatibility. The biomaterial components of prosthetic devices in contact with the bloodstream regardless of contact duration must be hemocompatible; they do not cause clinically significant blood-related adverse events such as 
thrombosis, hemolysis, platelet and complement activation. Mostly the artificial surfaces can induce clotting, and high levels of coagulation is associated with acute thrombosis or thromboembolism that determine the failure of biomaterials/device $(40,41)$. The hemocompatibility depends on the material characteristics but also on the fluid mechanics of the device and the blood coagulability. The key points in hemocompatibility testing are coagulation, hemolysis, hematology, platelets, and complement system. The assessment involves static, agitated or shear flow in vitro models for the incubation of fresh human blood with biomaterials. The hemocompatibility markers are determined before and after the incubation of the test material (42).

Coagulation. Coagulation is evaluated in vitro by measuring the rate of clot formation or the partial thromboplastin time (PTT) of plasma exposed to the biomaterials during an incubation time. A shortening of PTT induced by contact with biomaterials compared to the negative control shows the activation of the internal coagulation pathway. The amount of thrombin, the main enzyme of the coagulation pathway can also be determined by measuring thrombin-antithrombin complex (TAA) and prothrombin fragment $1+2$ using ELISA techniques. The TAA complex reflects a functional state of the coagulation, and prothrombin fragments are released during thrombin formation. An overall picture of the clotting process can be obtained by thromboelastography, an in vitro whole-blood viscoelastic test $(40,42)$.

Hemolysis analysis. Hemolysis analysis deals with the evaluation of the degree of erythrocyte lysis and the release of hemoglobin induced by the tested biomaterial. The determination of plasma hemoglobin is performed by spectrophotometric methods. Immunonephelometry and ELISA techniques may be other options. For device components having direct contact with the blood, hemolysis testing is recommended by both direct and indirect methods. Only an indirect method is indicated in the case of devices having indirect contact with circulating blood. In direct testing, the blood is incubated with biomaterial and in indirect method, the blood is exposed to the biomaterial extract. Depending on the hemolysis testing results, the material are classified as hemolytic (over 5\% hemolysis), slightly hemolytic (between 5 and 2\%) and nonhemolytic (below 2\%) $(40,42)$.

Hematology testing. Hematology testing includes the assessment of the complete blood count and the activation of leukocytes as a result of biomaterial-induced inflammatory response. Hematology analyzer and ELISA method are used in this direction. Since the activation of leukocytes leads to an enhanced oxygen metabolism, the excessive reactive oxygen species generation can be evaluated using fluorogenic or chemiluminogenic agents. In addition, the release of polymorphonuclear leukocyte elastase, an another event induced by leukocyte activation, can be quantified by ELISA or fluorescence $(40,42)$.

Platelet activation testing. Several points can be used for the evaluation of undesired platelet activation induced by the blood-biomaterial contact. They involve the quantification of degranulation proteins (platelet factor 4, b-thromboglobulin, thromboxane B2) released after platelet activation by ELISA, detection of P-selectin (CD62P) or activated GPIIb/IIIa using flow cytometry $(40,42)$.

Complement system activation. The complement system is an important component of the innate immune response and it is primarily involved in the first-line host defense against pathogenic factors through three major pathways (classical, alternative and lectin pathway). Biomaterials act primarily upon the alternative pathway of complement, and the complement activation is associated with the generation of complement proteins ( $\mathrm{C} 3, \mathrm{C} 5 \mathrm{~b}, \mathrm{C} 6, \mathrm{C} 7, \mathrm{C} 8, \mathrm{C} 9)$ that can be determined using ELISA. In addition, the complement activation can be analyzed by the evaluation of the $50 \%$ complement hemolytic activity (CH50). In general, the hydrophobic surfaces of biomaterials cause an increased complement activation compared to hydrophilic surfaces. The binding of complement components to the biomaterial surfaces can reduce their plasma content and can alter the final result $(40,42)$.

Implantation tests. Implantation tests evaluate the local pathological effects on living tissues that constitute the target of the biomaterial/medical device used for implantation. The biological response of the surrounding tissues (number and distribution of inflammatory cells, vascularity of fibrous capsule, granuloma, fatty infiltration, material debris, endothelialization, presence of necrosis) is commonly characterized by histological analysis. Both the tissue from the immediate vicinity of the implant and more distant tissues are studied.

In implantation tests, the safety of materials is assessed not only dose-dependently but also time-dependently. The tests are performed using mice, rats, guinea pigs or rabbits for short-term evaluation studies (12 weeks) (43-45). The rabbits are preferred for the musculoskeletal implantation studies since they have faster skeletal change, a high bone turn over and are less expensive and easy to handle (12). The animals with relatively long life expectancy such as dogs, sheep, goats, are used in longer-term studies. The chemistry, size and the degradation pattern of the materials significantly influence the biological response (43-45).

\section{Conclusions}

It is undeniable that the biocompatibility concept is a major concern in the design of biomaterials in orthopedics. A wide array of specific tests must be performed in order to ensure safety and efficacy of the biomaterials as this paper has reviewed. The development of new materials, the new paradigm of biocompatibility ('do no harm' approach is transformed into one of doing 'good') (5) and the understanding of the role of biomaterial surface physicochemistry accentuate the need for a calibration of regulatory criteria depending on new technologies and concepts. The design of biomaterials with high biocompatibility and functional properties (antimicrobial, osteoinductive) is highly desirable.

\section{Acknowledgements}

Not applicable. 


\section{Funding}

No funding was received.

\section{Availability of data and materials}

Not applicable.

\section{Authors' contributions}

$\mathrm{BH}$ wrote the initial draft of the manuscript, contributed to the conception and design of the article and performed the literature data collection. BP and RMN contributed to the design of the article. BP, RMN, SG, GP and AF consulted relevant references and performed the literature data collection. OA and PDS revised the manuscript in light of the literature findings. All authors have read and approved the final version of the manuscript for publication.

\section{Ethics approval and consent to participate}

Not applicable.

\section{Patient consent for publication}

Not applicable.

\section{Competing interests}

The authors declare that they have no competing interests.

\section{References}

1. Cieza A, Causey K, Kamenov K, Hanson SW, Chatterji S and Vos T: Global estimates of the need for rehabilitation based on the global burden of disease study 2019: A systematic analysis for the global burden of disease study 2019. Lancet 396: 2006-2017, 2021.

2. GBD 2019 Diseases and Injuries Collaborators: Global burden of 369 diseases and injuries in 204 countries and territories, 1990-2019: A systematic analysis for the global burden of disease study 2019. Lancet 396: 1204-1222, 2020.

3. Assad M and Jackson N: Biocompatibility evaluation of orthopedic biomaterials and medical devices: A review of safety and efficacy models. In: Encyclopedia of Biomedical Engineering. Vol 2. 1st edition. Narayan RJ (ed). Elsevier, Amsterdam, pp281-309, 2019.

4. Bruinink A and Luginbuehl R: Evaluation of biocompatibility using in vitro methods: Interpretation and limitations. Adv Biochem Eng Biotechnol 126: 117-152, 2012.

5. Helmus MN, Gibbons DF and Cebon D: Biocompatibility: Meeting a key functional requirement of next-generation medical devices. Toxicol Pathol 36: 70-80, 2008.

6. Bergmann CP and Stumpf A: Biomaterials. In: Dental ceramics. Topics in mining, metallurgy and materials engineering. Bergmann CP (ed) Springer, Heidelberg, pp9-15, 2013.

7. Rodríguez-González ÁF: Biomaterials in Orthopaedic Surgery. ASM International, pp1-10, 2009.

8. Shekhwat D, Singh A, Banerjee MK, Singh T and Patnaik A: Bioceramics composites for orthopaedic applications: A comprehensive review of mechanical, biological, and microstructural properties. Ceram Int 47: 3013-3030, 2021.

9. Kiradzhiyaska DD and Mantcheva RD: Overview of biocompatible materials and their use in medicine. Folia Med (Plovdiv) 61: 34-40, 2019.

10. Gobbi SJ, Gobbi JV and Rocha Y: Requirements for selection/development of a biomaterial. Biomed J Sci Tech Res 14: 10674-10679, 2019.

11. Navarro M, Michiardi A, Castaño O and Planell JA: Biomaterials in orthopaedics. J R Soc Interface 5: 1137-1158, 2008.
12. Vandana U, Nancy D, Sabareeswaran A, Remya NS, Rajendran N and Mohanan PV: Biocompatibility of strontium incorporated ceramic coated titanium oxide implant indented for orthopaedic applications. Mater Sci Eng B 264: 114954, 2021.

13. Bommala VK, Krishna MG and Rao CT: Magnesium matrix composites for biomedical applications: A review. J Magnes Alloy 7: 72-79, 2019.

14. Kumar K, Das A and Prasad SB: Recent developments in biodegradable magnesium matrix composites for orthopaedic applications: A review based on biodegradability, mechanical and biocompatibility perspective. Mater Today Proc 44: 2038-2042, 2021.

15. Nuss KMR and von Rechenberg B: Biocompatibility issues with modern implants in bone-a review for clinical orthopedics. Open Orthop J 2: 66-78, 2008

16. Victor SP and Muthu J: Polymer ceramic composite materials for orthopedic applications-relevance and need for mechanical match and bone regeneration. J Mechatron 2: 1-10, 2014.

17. Aherwar A, Singh AK and Patnaik A: Current and future biocompatibility aspects of biomaterials for hip prosthesis. AIMS Bioeng 3: 23-43, 2016.

18. Kattimani VS, Kondaka S and Lingameneni KP: Hydroxyapatitepast, present, and future in bone regeration. Bone Tissue Regen Insights: Sep 11, 2016 (Epub ahead of print).

19. Rufino Senra M and Marques FV: Synthetic polymeric materials for bone replacement. J Compos Sci 4: 191, 2020.

20. Liu B and Lun DX: Current application of $\beta$-tricalcium phosphate composites in orthopaedics. Orthop Surg 4: 139-144, 2012.

21. Samavedi S, Poindexter LK, Van Dyke M and Goldstein AS: Synthetic biomaterials for regenerative medicine applications. In: Regenerative Medicine Applications in Organ Transplantation. Orlando G, Lerut J, Soker S and Stratta RJ (eds). Academic Press, Boston, pp81-99, 2014.

22. Webb JC and Spencer RF: The role of polymethylmethacrylate bone cement in modern orthopaedic surgery. J Bone Joint Surg Br 89: 851-857, 2007.

23. Cipurković A, Horozić E, Đonlagić N, Marić S, Saletović M and Ademović Z: Biodegradable polymers: Production, properties and application in medicine scientific review paper. Technol Acta 11: 25-35, 2018.

24. Quinn J, McFadden R, Chan CW and Carson L: Titanium for orthopedic applications: An overview of surface modification to improve biocompatibility and prevent bacterial biofilm formation. iScience 28: 10174, 2020.

25. Rahmati M, Silva EA, Reseland JE, Heyward CA and Haugen HJ: Biological responses to physicochemical properties of biomaterial surface. Chem Soc Rev 49: 5178-5224, 2020.

26. Cvrček L and Horáková M: Plasma modified polymeric materials for implant applications. In: Non-Thermal Plasma Technology for Polymeric Materials. Applications in Composites, Nanostructured Materials and Biomedical Fields. Thomas S, Mozetič M, Cvelbar U, Špatenka P and Praveen KM (eds). Elsevier, Amsterdam, pp376-407, 2019.

27. Groth T, Falck P and Miethke RR: Cytotoxicity of biomaterials-basic mechanisms and in vitro test methods: A review. ATLA 23: 790-799, 1995.

28. Iqbal HMN and Keshavarz T: The challenge of biocompatibility evaluation of biocomposites. In: Woodhead Publishing Series in Biomaterials, Biomedical composites (second edition). Ambrosio L (ed). Woodhead Publishing, Cambridge, pp303-334, 2017.

29. Li W, Zhou J and Xu Y: Study of the in vitro cytotoxicity testing of medical devices. Biomed Rep 3: 617-620, 2015.

30. Kohl Y, Rundén-Pran E, Mariussen E, Hesler M, El Yamani N, Longhin EM and Dusinska M: Genotoxicity of nanomaterials: Advances in vitro models and high throughput methods for human hazard assessment-a review. Nanomaterials (Basel) 10: 1911,2020

31. Raghavendra GM, Varaprasad $\mathrm{K}$ and Jayaramudu $\mathrm{T}$ : Biomaterials: Design, development and biomedical applications. In: Nanotechnology Applications for Tissue Engineering. Thomas S, Grohens Y and Ninan N (eds). Elsevier, Amsterdam, pp21-44, 2015.

32. Moore MM, Honma M, Clements J, Bolcsfoldi G, Burlinson B, Cifone M, Clarke J, Delongchamp R, Durward R, Fellows M, et al: Mouse lymphoma thymidine kinase gene mutation assay: Follow-up meeting of the international workshop on genotoxicity testing-aberdeen, scotland, 2003-assay acceptance criteria, positive controls, and data evaluation. Environ Mol Mutagen 47: 1-5, 2006. 
33. OECD Guideline for the Testing of Chemicals. In vitro mammalian cell gene mutation assays using the thymidine kinase gene, 2014. Availablefrom:https://www.oecd.org/env/ehs/testing/In\%20Vitro\% 20Mammalian\%20Cell\%20Gene\%20Mutation\%20Thymidine\% 20Kinase\%20.pdf.

34. OECD/OCED Guideline for the Testing of Chemicals. In vitro mammalian chromosomal aberration test, 2016. Available from: https://www.oecd-ilibrary.org/docserver/9789264264649-en.pdf? expires $=1615635244 \& \mathrm{id}=\mathrm{id} \&$ accname $=$ guest $\&$ checksum $=\mathrm{AD} 49$ 4BA2A538E6C1302075C64D4C7BA5.

35. Registre $\mathrm{M}$ and Proudlock R: The in vitro aberration test. In: Genetic toxicology testing. Proudlock R (ed). Academic Press, Amsterdam, pp207-267, 2016.

36. Jain AK, Singh D, Dubey K, Maurya R, Mittal S and Pandey AK Models and methods for in vitro toxicity. In: In vitro Toxicology. Dhawan A and Kwon S (eds). Academic Press, Amsterdam, pp45-65, 2018.

37. Guy RC: Ames test. In: Encyclopedia of Toxicology. 2nd edition. Wexler P (ed). Elsevier, Amsterdam, pp88-91, 2005.

38. De Jong WH, Caraway JW and Geertsma RE: In vivo and in vitro testing for the biological evaluation of biomaterials and medical devices. In: Woodhead Publishing Series in Biomaterials, Biocompatibility and Performance of Medical Devices. Boutrand JP (ed). Woodhead Publishing, Cambridge, pp120-158, 2012.

39. Kimber I, Basketter DA, Berthold K, Butler M, Garrigue JL, Lea L, Newsome C, Roggeband R, Steiling W, Stropp G, et al: Skin sensitization testing in potency and risk assessment. Toxicol Sci 59: 198-208, 2001.
40. Nalezinková M: In vitro hemocompatibility testing of medical devices. Thromb Res 195: 145-190, 2020.

41. Brănisteanu DE, Nichifor M, Dorobăt CM, Brănisteanu DC, Petrariu FD, Molodoi AD, Radu DC and Boda D: Use of textile biomaterials for the topic treatment of chronic venous disease. Rom Biotechnol Lett 20: 10618-10625, 2015.

42. Weber M, Steinle H, Golombek S, Hann L, Schlensak C, Wendel HP and Avci-Adali M: Blood-contacting biomaterials: In vitro evaluation of the hemocompatibility. Front Bioeng Biotechnol 6: 99, 2018.

43. Anderson JM and Schoen FJ: In vivo assessment of tissue compatibility. In Biomaterials Science. 3rd edition. Ratner BD, Hoffman AS, Schien FJ and Lemons JE (eds). Academic Press, Amsterdam, pp609-617, 2013.

44. Anderson JM and Jiang S: Animal models in biomaterial development. In: Encyclopedia of biomedical engineering. Narayan R (ed). Elsevier, Amsterdam, pp237-241, 2019.

45. Hakimi O, Vollrath F and Carr AJ: Evaluation of silk as a scaffold for musculoskeletal regeneration-the path from the laboratory to clinical trials. In: Comprehensive biotechnology. 2nd edition. Moo-Young M (ed). Academic Press, Amsterdam, pp341-351, 2011.

This work is licensed under a Creative Commons Attribution-NonCommercial-NoDerivatives 4.0 International (CC BY-NC-ND 4.0) License. 